\title{
Adapting Requirements Models to Varying Environments
}

\author{
Dalal Alrajeh \\ Department of Computing, Imperial \\ College London \\ UK \\ dalal.alrajeh@ic.ac.uk
}

\author{
Antoine Cailliau \\ ICTEAM, UCLouvain \\ Belgium \\ antoine.cailliau@uclouvain.be
}

\author{
Axel van Lamsweerde \\ ICTEAM, UCLouvain \\ Belgium \\ axel.vanlamsweerde@uclouvain.be
}

\begin{abstract}
The engineering of high-quality software requirements generally relies on properties and assumptions about the environment in which the software-to-be has to operate. Such properties and assumptions, referred to as environment conditions in this paper, are highly subject to change over time or from one software variant to another. As a consequence, the requirements engineered for a specific set of environment conditions may no longer be adequate, complete and consistent for another set.

The paper addresses this problem through a tool-supported requirements adaptation technique. A goal-oriented requirements modelling framework is considered to make requirements' refinements and dependencies on environment conditions explicit. When environment conditions change, an adapted goal model is computed that is correct with respect to the new environment conditions. The space of possible adaptations is not fixed a priori; the required changes are expected to meet one or more environment-independent goal(s) to be satisfied in any version of the system. The adapted goal model is generated using a new counterexample-guided learning procedure that ensures the correctness of the updated goal model, and prefers more local adaptations and more similar goal models.
\end{abstract}

\section{CCS CONCEPTS}

- Software and its engineering $\rightarrow$ Requirements analysis; Software evolution; Model-driven software engineering.

\section{KEYWORDS}

Requirements adaptation, requirements evolution, context-dependent requirements, formal verification, logic-based learning

\section{ACM Reference Format:}

Dalal Alrajeh, Antoine Cailliau, and Axel van Lamsweerde. 2020. Adapting Requirements Models to Varying Environments. In Proceedings of ACM Conference (Conference'17). ACM, New York, NY, USA, 12 pages. https: //doi.org/10.1145/nnnnnnn.nnnnnnn

\section{INTRODUCTION}

The engineering of adequate, consistent and complete software requirements is known to rely on properties and assumptions about

Permission to make digital or hard copies of all or part of this work for personal or classroom use is granted without fee provided that copies are not made or distributed for profit or commercial advantage and that copies bear this notice and the full citation on the first page. Copyrights for components of this work owned by others than ACM must be honored. Abstracting with credit is permitted. To copy otherwise, or republish, to post on servers or to redistribute to lists, requires prior specific permission and/or a fee. Request permissions from permissions@acm.org.

Conference'17, July 2017, Washington, DC, USA

(C) 2020 Association for Computing Machinery.

ACM ISBN 978-x-xxxx-xxxx-x/YY/MM...\$15.00

https://doi.org/10.1145/nnnnnnn.nnnnnnn the environment in which the software will operate $[29,40]$. In particular, lower-level requirements conjoined with such properties and assumptions must entail the higher-level requirements to which they contribute [49]. The documentation of requirements satisfaction arguments showing this is an important aspect of the requirements engineering (RE) process [29, 36], notably for traceability management and requirements verification purposes [48].

Properties and assumptions about the environment, called environment conditions hereafter, are subject to change over time and space-as the system evolves over time or as system variants are being considered over space. The originally engineered requirements may no longer be adequate, consistent nor complete when those properties and assumptions change. As a consequence, the original requirements must be adapted to ensure their adequacy, consistency and completeness in the new environment.

This paper uses a goal-oriented RE framework in order to make requirements refinements and dependencies on environment conditions fully explicit [48]. To illustrate the problem addressed in the paper, consider an example of an urban traffic control system aimed at enforcing low emission zones. Air pollution is estimated to be responsible for 310,000 premature deaths in Europe each year, causing more of these than road accidents [2]. Low Emission Zones (LEZs) are urban areas where the most polluting vehicles are regulated. Consider a software house developing LEZ enforcement software for cities across Europe [44]. A high-level goal for such system is that "urban traffic death toll shall be reduced". To achieve this, the goal is refined by prescribing the subgoal "G: polluting vehicles shall be penalized", among others. When developing the London product, the latter goal is refined into subgoal "SG: polluting vehicles shall be charged when entering designated LEZ", among others. The latter goal depends on a number of environment conditions, e.g., being in London where polluting vehicles are admitted within such zone, but must pay a charge of $£ 22$. For cities like Brussels, Frankfurt and Stockholm, the higher-level goal $G$ remains the same in spite of different environment conditions. For Brussels, in particular, the London environment conditions do not hold since polluting vehicles are not admitted in LEZ in Brussels; the lower-level London goal $S G$ thus no longer makes sense; it must be adapted to match the Brussels' regulations (e.g., by issuing fines). The problem for the software RE team gets exacerbated as regulations are not only varying over space (across different countries and cities) but also over time as regulations within the same city evolve in unpredictable ways (in Brussels, they changed once over the last two years). Advanced vehicle technologies (e.g., electric cars) are also expected to call for other types of system evolutions. 
Assume a LEZ enforcement software product is developed for London. Options for delivering this product to other cities worldwide would currently include: $(a)$ adapt the software implementation directly regardless of the London requirements-the resulting implementation would then be requirements-free, or inconsistent with the original requirements, making it impossible to enable requirements-based testing, traceability management, and so forth; (b) develop a set of business rules, or a feature diagram along a product line approach [17], for deciding which requirements to consider in which city; this appears highly unfeasible given the huge variation space, its expansion over time, and the unpredictability of evolving city regulations; (c) manually adapt the London requirements to match the specifics of each city-a costly and error-prone task given for similar reasons and the interdependency among requirements; or $(d)$ provide automated support for the requirements adaptation process-as we propose in this paper.

The requirements adaptation problem can be more precisely defined in terms of the well-known RE reference framework [49] transposed to goal models [48]. GIVEN: $(i)$ a set of system goals $G$; a set of lower-level subgoals $S G$ contributing to $G$; one or more common, environment-independent goals $C G$, to be found in any system variant or evolution, to which $G$ contributes; a set of environment conditions $E$ such that $\{S G, E\} \mid=G$ and $\{G, E\} \mid=C G$ (where $=$ denotes logical entailment), (ii) an environment variation $E^{\prime}$ of $E$ compatible with the preservation of the original high-level common goal(s) $C G$; FIND adapted goals $G^{\prime}$ and lower-level subgoals $S G^{\prime}$ where $\left\{S G^{\prime}, E^{\prime}\right\} \mid=G^{\prime}$ and $\left\{G^{\prime}, E^{\prime}\right\} \mid=C G$. The new environment conditions should thus allow one or more common high-level goals to remain stable through all system evolutions or variants; it would not make much sense to consider ones leading to an adapted system having no common purpose with the original.

Little research attention has been paid to provide automated support for this adaptation problem. Much of RE work on adaptation so far focuses on reconfiguration within an adaptation space known in advance [3, 4, 9, 14, 31, 34]. The preservation of requirements satisfaction arguments under environment changes is never addressed there. Work on requirements variants in static or dynamic product lines also assumes the space of all possible variations to be known a priori (e.g., in a feature diagram) [26, 27]. A significant amount of work on requirements evolution has been devoted to requirements traceability and requirements organization for easier change management [30]. The work closest to ours is [8] where a learning technique for revising goal models is also described. The problem in [8] is, however, different and the solution there is not adequate for the adaptation problem considered here. In [8], environment conditions are not changing and assumed to be correct; new conditions may be added but none removed. Preservation of goal model correctness w.r.t. higher-level goals is not considered. In contrast, our aim here is to generate adaptations that preserve model correctness across varying environments. Additionally, the learning technique there can only modify goal specifications; the goal model structure remains fixed. Our adaptation problem may require other types of changes (such as changing the refinement structure). Finally, the learning technique in [8] does not yield revisions guaranteed to be local and similar to the original model In [14], predefined sets of countermeasures to goals obstructions are dynamically selected at system runtime to reduce the monitored obstruction rates. Note that our requirements adaptation problem might sometimes be addressed at system runtime, runtime adaptations are, however, not discussed here.

This paper provides a formal, tool-supported approach to address the adaptation problem defined above at system development time. For this purpose, we use the KAOS goal-oriented RE framework [48] to model requirements and reason about their adaptations. This framework is chosen because: $(i)$ requirements dependencies on other requirements and on environment conditions is made fully explicit; (ii) multiple abstraction levels are supported; (iii) requirements can be formally specified, enabling the use of formal analysis tools; and (iv) the modelling framework has been used in industry [19], e.g., for Huawei smartphones [41]. Thus given a source goal model, the proposed approach computes model adaptations for a different target environment where:

- The adaptations may cover model evolution over time or model variants over space;

- The space of variations is not explicitly known in advance; it is, however, constrained by the preservation of environmentindependent goals to be commonly met across adaptations;

- The approach preserves model correctness; it is driven by satisfaction arguments of the form $\left\{S G^{\prime}, E^{\prime}\right\} \mid=G^{\prime}$ and $\left\{G^{\prime}, E^{\prime}\right\} \mid=C G$, given the original model where $\{S G, E\} \mid=G$ and $\{G, E\} \mid=C G$.

At the heart of our solution is a novel counterexample-guided learning technique for adapting requirements expressed in metric linear temporal logic (MLTL [32]). In brief, given a correct goal model where the source, original environment conditions are replaced with the target, new ones, our procedure verifies the correctness of the refinements of each goal in the model. If the verification finds an incorrect refinement, counterexamples demonstrating this are automatically generated. These traces are then used as examples by a logic-based learning procedure to revise goals appearing in the refinements so as to eliminate this violation. The procedure iterates until an updated goal model is obtained that fulfils the satisfaction arguments for every goal refinement in the model.

A key aspect of our technique is its ability to search for adaptations of an existing goal model that best meet some preference criteria. Our technique generates adaptations to goals and their refinements that are syntactically as close as possible to the original ones, with the fewest goals being adapted, whilst ensuring the model's correctness. The paper makes the following contributions: - a dedicated notion of context and context-dependent property for reasoning about dependencies on changing environment conditions in the RE process;

- a novel adaptation method for formal goal models at development time that is guaranteed to produce "minimal" adaptations;

- a software tool that integrates model checking and logic-based learning with preferences; our implementation supports multiple degrees of automation (from fully automated mode to semiautomated mode allowing for human intervention to guide the search towards relevant adaptations);

- an evaluation of the correctness and the applicability of our approach on real adaptation problems. We particularly show how adaptations to goal specifications, goal refinement tactics and goal refinement depths are effectively computed. 
The paper is organized as follows. Section 2 recalls some required material on goal modelling and logic-based learning. Section 3 reviews the sources of variability in goal models. Section 4 defines the requirements adaptation problem. Section 5 gives an overview of our solution. Section 6 details the adaptation procedure. Section 7 discusses our tool implementation. Section 8 evaluates the approach with respect to correctness and applicability. We discuss aspects of scalability and human intervention in Section 9. Section 10 reviews related work while Section 11 concludes our work.

\section{BACKGROUND}

\subsection{Goal-oriented system modelling}

In KAOS [48], a goal is a prescriptive statement of intent to be satisfied by cooperation of the agents forming the system. The word system refers to both the software and its environment, including people, legacy software, devices like sensors and actuators, etc. An agent is an active system component having responsibilities in goal satisfaction and capabilities in terms of conditions the agent can monitor or control. A environment property is a descriptive statement about the system, e.g., a physical law or a regulation.

We focuse on behavioural goals whose satisfaction is determined in a clear-cut sense [48]. A behavioural goal defines a maximal set of behaviours declaratively. A behaviour violates a goal if it is not among the behaviours prescribed by it. To enable formal analysis, behavioural goals are specified in a metric linear temporal logic (MLTL). The syntax of MLTL formulae is defined over a finite nonempty set of propositional variables, the logical constants true and false, the standard Boolean connectives and the temporal operators $\bigcirc$ (next state), $\diamond$ (sometime in the future), $\diamond_{\leq d}$ (sometime in the future before deadline $d$ ), $\square$ (always in the future), and $\square_{\leq d}$ (always in the future up to deadline $d$ ). The formulae $p$ and $\neg p$, where $p \in \mathcal{P}$, are called positive and negative literals, respectively.

A behavioural goal $G$ is of type Achieve or Maintain. The specification pattern for Achieve goals is $\square($ Current $\rightarrow \Theta$ Target) or Current $\Rightarrow$ $\Theta$ Target for short (as in [37]), where Current- and Target-conditions are Boolean formulae, and $\Theta \in\left\{\bigcirc, \diamond_{\leq d}, \diamond_{<d}\right\}$. The pattern for Maintain goals is $\square$ (Current $\rightarrow$ Target $)$, shortened to Current $\Rightarrow$ Target. We refer to behavioural goals written in MLTL as goal specifications. Their semantics is defined over infinite sequences of states in the standard way [33]. Each state $s_{i}$ is uniquely identified by the valuation of variables. We consider lasso-shaped traces of the form $\sigma=w_{1}\left(w_{2}\right)^{\omega}$, where $w_{1}=s_{1} s_{2} \ldots$ and $w_{2}=s_{k} s_{k+1} \ldots$ are finite sequences [12]. We use $\sigma(j)$ to denote the $j$ th state of $\sigma$.

A goal model is an AND/OR graph showing how goals are refined, and how their responsibilities are assigned to system agents. An AND-refinement defines a set of subgoals and environment conditions that together entail the parent goal. An OR-refinement captures an alternative AND-refinement. Leaf goals of the AND/OR graph are assigned as responsibilities to single agents. A goal assigned to a software agent is a software requirement whereas a goal assigned to an environment agent is an environment assumption. As a precondition for assigning a leaf goal to an agent, the goal must be realizable by the agent, that is, it must have finite bounds in case of an Achieve goal, and the propositional variables in the Target-part of the goal specification must be controllable by the agent whereas the propositional variables in the Current-part must

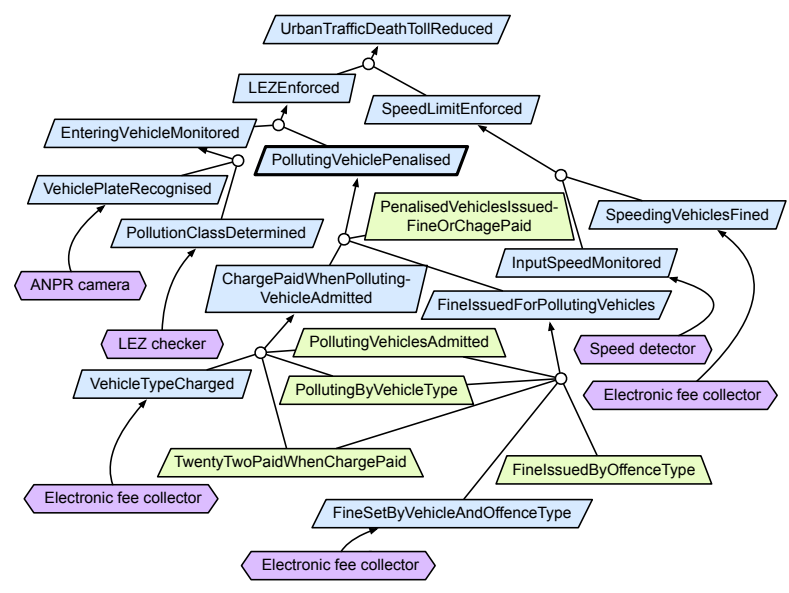

Figure 1: Goal model for London's low emission zone.

be monitorable by it [48]. An agent's capabilities are defined by the sets of propositional variables it can monitor and control.

A goal is defined in terms of a name, type, its refinement, a formal specification and an agent assignment for leaf goals. An environment condition is defined by a name and a formal specification. The notation $G_{\mathbf{X}}$, where $\mathbf{X} \in\{$ Type, RefinedBy, Spec, $\operatorname{Resp}\}$, refers to specific components of a goal definition, and similarly for environment conditions. $G \backslash\left\{\mathbf{X}_{i}\right\}$ means the goal definition excluding the components in $\left\{\mathbf{X}_{i}\right\}$. A goal model is a collection of goal and environment condition definitions. It is captured diagrammatically with goals, environment conditions and agents represented by parallelograms, trapezes and hexagons, respectively. Edges connected by a white circle capture an AND-refinement link. Directed edges from hexagons capture agent assignments. Fig. 1 shows a goal model fragment for the LEZ example. The root goal is AND-refined into two subgoals which are in turn further AND-refined. The leaf goal VehicleTypeCharged is assigned to the ElectronicFeeCollector agent.

A goal model is correct if all its AND-refinements are complete, consistent and minimal. An AND-refinement of a parent goal $P G$ into subgoals $\left\{S G_{1}, \ldots, S G_{n}\right\}$ is complete if the subgoals $\left\{S G_{i}\right\}$ and environment conditions $E$ are sufficient to satisfy $P G$ :

$$
\left\{S G_{1}, \ldots, S G_{n}, E\right\} \mid=P G \quad \text { (complete refinement) }
$$

The refinement is consistent if the subgoals are consistent with the environment conditions:

$$
\left\{S G_{1}, \ldots, S G_{n}, E\right\} \not \text { false (consistent refinement) }
$$

The refinement is minimal if it contains a minimal set of subgoals, $\left\{S G_{i}\right\}$, necessary to satisfy the parent goal $P G$ :

$$
\left\{\bigwedge_{k \neq j} S G_{k}, E\right\} \not \models P G \quad \text { (minimal refinement) }
$$

where we represent a conjunction of conjuncts as a set of conjuncts for convenience.

\subsection{Logic-based Learning}

Logic-based learning is a symbolic machine learning technique concerned with inductively inferring general logical theories that, with a given background knowledge, explain a given set of examples [38]. State-of-the-art tools, referred to as non-monotonic logic learning systems, are capable of learning generalizations from examples, and 
revisions of existing logical theories [11, 18] expressed as normal logic programs (i.e., logic programs with negation as failure) [35].

A revision task is a tuple $\left\langle B, O^{+}, O^{-}, K\right\rangle$ where $B$ is a correct logical theory (a set of clauses), called the background theory, $\mathrm{O}^{+}$and $\mathrm{O}^{-}$ are sets of atomic formulae called positive and negative observations (a.k.a. examples) respectively and $K$ is a possibly incorrect logical theory, called the revisable theory, such that $B$ and $K$ do not cover all examples in $\mathrm{O}^{+}$and/or do not exclude all examples in $\mathrm{O}^{-}$; formally $O^{+} \nsubseteq \mathbb{M}(B \wedge K)$, where $\mathbb{M}(B \wedge K)$ denotes the semantic model of $B \wedge K$, and $O^{-} \cap \mathrm{M}(B \wedge K) \neq \emptyset$. Typically the semantic model $\mathrm{M}(\phi)$ is the set of atomic formulae that follow from $\phi$.

An inductive solution to such task is a revised theory $\widetilde{K}$ that, together with $B$, covers all examples in $O^{+}$, that is, $O^{+} \subseteq \mathbb{M}(B \wedge \widetilde{K})$ and excludes all negative ones $O^{-}$, i.e., $O^{-} \cap \mathrm{M}(B \wedge \widetilde{K})=\emptyset$. The computation of $\widetilde{K}$ is typically achieved through a transformation function that applies change operations (adding/deleting literals or clauses) to $K$. To make the problem tractable, the search for revisions is generally performed within the scope of a search space defined by a mode bias $I$, that reduces the set of candidate solutions by restricting the set of literals that can be added to or removed from the revisable theory $K$. Further restrictions can be imposed by declaring structural constraints $D$ on the set of logical expressions that may be included within the solution space (see [10] for details). In this setting, a revision task is now defined as a tuple $\left\langle B, O^{+}, O^{-}, K, I, D\right\rangle$. A logical theory $\widetilde{K}$ is an acceptable revision if and only if $(a)$ it is an inductive solution; $(b)$ it is is within the scope of the search space defined by the mode bias $I$; and (c) it satisfies all structural-constraints $D$. Recent state-of-the-art learning systems (as the one used in this paper) implement a revision task as a satisfiability problem for which efficient solvers exist [22].

Typically, logic-based learning algorithms generate minimal solutions, i.e., ones with the fewest number of clauses and literals. When learning revisions, minimal solutions are ones whose generation involves the smallest number of change operations. This enables the revision task to search for solutions that, for instance, minimize the sum of weights of the derivable consequences in the highest priority level, and then those in the next lower level, and so forth. A solution is minimal if the sum of weights of atomic formulae that hold is maximal or minimal, as required by the statement, among all semantic models of $B \wedge \widetilde{K}$.

\section{ENVIRONMENT CONDITIONS IN CHANGING CONTEXTS}

As introduced in Section 1, environment conditions are properties and assumptions about the environment in which the software is intended to operate. They can take one of three forms: (i) descriptive properties of the environment: these are the environment properties introduced in Section 2 (e.g., the trapeze PollutingVehiclesAdmitTED in Fig. 1); (ii) prescriptive assumptions about the environment: these are the goals assigned to environment agents as introduced in Section 2 (e.g., the leaf goal VehiclePlateRecognized assigned to the ANPR camera agent in Fig. 1); and (iii) descriptive assumptions about the environment: these are often implicitly assumed and are needed for refinements to be correct (e.g., Vehicle $\Leftrightarrow$ Car $\vee$ Lorry $\vee$ Bus).

Environment conditions may hold in specific "contexts" only, e.g., PollutingVehiclesAdmitted holds only in the London context but not in the Brussels one. A context is defined as a set of monitorable facts that are subject to change over time or space, and that restrict the validity of an environment condition. A context has a name, called context label, (e.g., London) and a context predicate (e.g., InLondon). In the general case, a context may be specified in disjunctive normal form where each literal corresponds to a monitorable fact, e.g., InLondon $\vee$ InBrussels. Contexts form a Boolean lattice under $\vee-$ and $\wedge$ - operators with false and true as bottom and top, respectively. We can thus consider environment conditions whose validity spans over unions or intersections of contexts.

A context-dependent environment condition holds only in a restricted (set of) context(s). It is written as $C: \psi$ for "environment condition $\psi$ holds only in context labelled C", e.g., London : Polluting $\Rightarrow$ Admitted and Brussels : Polluting $\Rightarrow \neg$ Admitted.

A universal environment condition holds in all contexts. It corresponds to True : $\psi$ where True represents the set of all possible context labels. A context-dependent environment condition $C: \psi$ can be made universal through an implication $P_{C} \Rightarrow \psi$, where $P_{C}$ is the context predicate specifying the context labelled by $C$. In practice, we may wish to avoid this for a simpler formulation. An environment condition valid in a set of contexts is valid in each member context: if $C_{1}, \ldots, C_{n}: \psi$ then $C_{i}: \psi$. We write $E_{C} \subseteq E$ for the subset of environment conditions in $E$ dependent on $C$.

An AND/OR graph may integrate context-specific goal model adaptations as alternative OR-refinements. This enables moving back to previously considered contexts. It is, therefore, convenient to annotate goal refinements involving a context-dependent environment condition with the label of the context in which this condition is valid. Context-dependent refinements are thereby captured, as in $[5,48]$. In Fig. 1, the three bottom AND-refinements are context-dependent and should be annotated with the context label London. This label propagates up to a common OR-node to be created when an alternative context labelled Brussels is introduced.

\section{THE REQUIREMENTS ADAPTATION PROBLEM}

Changing contexts may invalidate the context-dependent environment conditions within a goal model. Hence a new goal model has to be computed that accounts for the environment conditions of the new context while preserving model correctness. There might be several model adaptations whose "qualities" differ. We identify here two qualities for desirable adaptations.

Similarity. It appears highly desirable to favour model adaptations that maintain similar functionalities across different contexts. We measure similarity between a goal $G$ and its adaptation $\widetilde{G}$ in terms of their respective types, refinements, formal specifications and agent assignments. More precisely, the similarity measure for these two goals is determined by summing up the number of definitions that are common to the two, and then deducting the number of definitions that are specific to $G$ and those that are specific to $\widetilde{G}$, as commonly done in analogical reasoning [47]. The similarity measure for two goal models is the sum of the similarity measures of their corresponding goals.

Locality. The impact of an adaptation can range from being local (e.g., affecting goals assigned to a single agent) to system-wide (e.g., affecting goals involving multiple agents). Adaptations brought 
about by changes that are localized to parts of a goal model are preferred over adaptations made of disperse changes across the whole goal model. This principle is inspired by the software evolution literature where disperse changes risk introducing new errors [13]. In our case, disperse changes to a goal model may lead to new incorrect refinements. To measure locality of an adaptation we introduce the notion of distance between connected goals in a goal model as the total number of refinement links between them. We say an adaptation $\widetilde{M 1}$ of $M$ is more local than $\overparen{M 2}$ of $M$ if the sum of the distances of all adapted goals in $\widetilde{M 1}$ is smaller than in $\widetilde{M 2}$.

To constrain the space of admissible adaptations to local adaptations and similar goal models, we introduce the concept of anchor goals [15]. Here, an anchor goal in a goal model is a non-leaf goal that should, together with its ancestors, remain unchanged in any adapted version of the model. In the LEZ example, for instance, an anchor goal should be PollutingVehiclesPenalized since, in any foreseeable context for LEZ enforcement, vehicles categorized as polluting are expected to be penalized even though how this goal is realized might vary from one context to another. To favour similarity among models and locality of adaptations, anchor goals should be selected at lowest possible levels in the model to be adapted whilst being compatible with the new context. (An anchor goal whose refinement is correct in context $C_{1}$ is said to be compatible with a context $C_{2}$ if it has a correct refinement in context $C_{2}$.)

Our adaptation task can be described more precisely as follows: GIVEN an adaptation problem $\left\langle E, C, M, C^{\prime}, \mathcal{A}, N\right\rangle$ where $E$ are the environment conditions $C$ is a source context; $M$ is a source goal model that is correct under $C$ according to properties (1)-(3) in Section 2.1; $C^{\prime}$ is a target context; $\mathcal{A}$ is a set of anchor goals in $M$; and $N$ are agents' capabilities specifications;

FIND a target goal model $\widetilde{M}$ adapted from $M$ such that: $\widetilde{M}$ is correct under the target context $C^{\prime}$; each anchor goal $A G \in \mathcal{A}$ is unchanged in $\widetilde{M}$; every leaf goal $\widetilde{L G}$ in $\widetilde{M}$ is realizable; $\widetilde{M}$ is a maximally local adaptation of $M$; and $\widetilde{M}$ is maximally similar to $M$.

\section{GOAL MODEL ADAPTATION: OVERVIEW}

Fig. 2 shows the basic workflow of our approach, called RESPIRE, for REquirementS adaPtation In vaRying Environments. Thickbordered input boxes are mandatory. At development time, the analyst provides: (i) an AND/OR graph $\mathcal{M}$, (ii) a set $\mathcal{A}$ of anchor goals ${ }^{1}$, (iii) a source context $C,\left(\right.$ iv) a target context $C^{\prime}$ and its environment conditions $E_{C^{\prime}}$, and $(v)$ the agents' capabilities specification $N$ for the target context ${ }^{2}$. We assume context names correctly label the corresponding context-dependent refinements in $\mathcal{M}$, each refinement is correct and the source context $C$ is indicated in $\mathcal{M}$.

Step 1 in Fig. 2, Acquire contextual changes, checks if $\mathcal{M}$ already contains a model variant, through its OR-branches, that is labelled with the target context $C^{\prime}$. If found, no adaptation is required and the model variant $\widetilde{M}$ is returned as the target goal model.

If the target model is not in $\mathcal{M}$, Extract reference model (Step 2 in Fig. 2) computes, from $\mathcal{M}$ and the source context, a reference goal model to be used for the adaptation process. This model comprises all AND-refinements that were labelled with the source context $C$

\footnotetext{
${ }^{1}$ If empty, RESPIRE assumes every root goal in $\mathcal{M}$ to be an anchor goal.

'If no agents' capabilities specification is provided, then the realizability condition is dropped from the task specification.
}

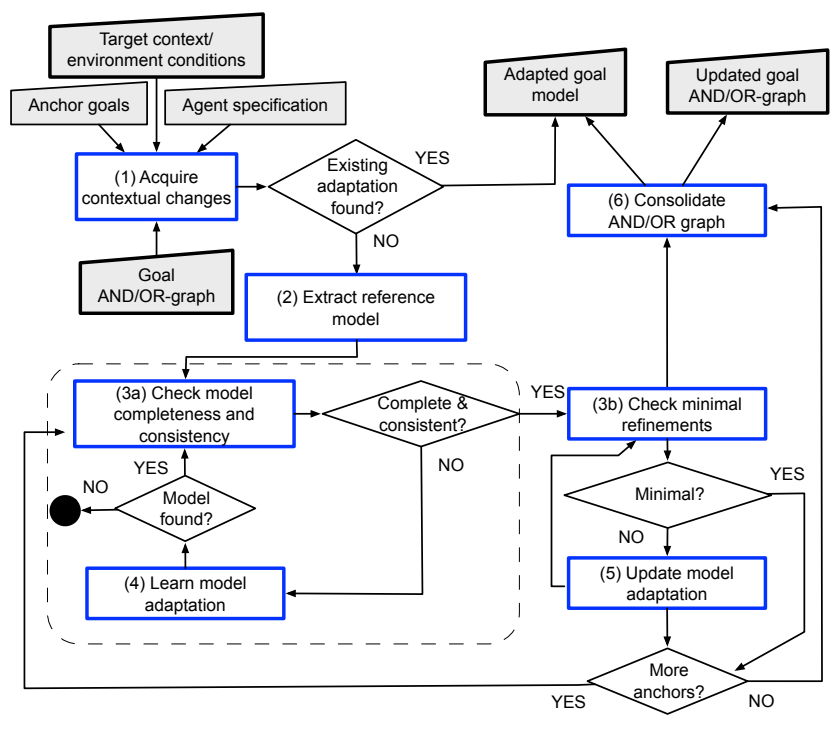

Figure 2: RESPIRE workflow.

but in which all environment conditions of the source context are replaced by those from the target context. This step defines a new adaptation problem as described in Section 4. RESPIRE then first a goal $A G \in \mathcal{A}$ (according to some assumed selection rule, in our case, the left most anchor goal appearing in the graph) and uses it to guide a two-stage adaptation process described below; the two stages are sequentially applied to each anchor goal in this model.

The first stage involves a counterexample-guided learning loop (enclosed in dashed lines in Fig. 2). The following steps are repeatedly called, starting from the reference goal model:

- Step 3a: Check model completeness and consistency under the target context. This involves: (i) constructing completeness and consistency properties given the selected anchor goal $A G$; (ii) generating counterexamples that violate the completeness and consistency properties under the target context; and (iii) generating witness examples to the satisfiability of the completeness and consistency properties under the target context;

- Step 4: Learn goal model adaptation of the reference goal model so that refinements in the adapted model are violated in the counterexamples, but hold in the witness examples;

- Go to step $3 a$.

The loop repeats until all refinements of the selected anchor goal $A G$ are complete and consistent in the adapted goal model, or the learner fails to find such a model. (The latter may occur owing to incompatibilities between the anchor goal and some new environment conditions.) If the refinements are complete and consistent with respect to the anchor goal $A G$, then the second stage of the adaptation is triggered.

This second stage involves checking that the refinements of $A G$ in the model generated from the first stage satisfy the minimality property in the Check minimal refinements process (Step $3 b$ in Fig. 2). If not satisfied, then Update model adaptation (Step 5 in Fig. 2) removes refinement links until all refinements satisfy it.

If there remain anchor goals in $\mathcal{A}$ that have not been checked for correctness, RESPIRE chooses one and instigates another iterative adaptation process as described above (Steps $3 a, 4,3 b$ and 5). The 
iteration terminates once the refinements of all anchor goals are correct under the target environment conditions considered, or no correct goal model adaptation is found by the learner.

If successful in finding a correct model adaptation, the procedure proceeds to Consolidate AND/OR-graph (Step 6 in Fig. 2), where the original graph AND/OR $\mathcal{M}$ is updated with an OR-refinement representing the computed target model. The output of RESPIRE is the newly computed target model and the updated $\mathcal{M}$ graph.

RESPIRE can run with varying degrees of human intervention within the dashed lines in Fig. 2, e.g., for leaf goal selection or for acceptance, rejection or modification of generated adaptation traces (see discussions in Sections 7 and 8).

\section{THE RESPIRE PROCEDURE}

This section details the core steps $3 a, 4,3 b$ and 5 in Fig. 2. We start with a reference model, $M_{C^{\prime}}^{0}$, in which all context-dependent environment conditions are now those of $C^{\prime}$, and anchor goals are labelled as anchors. In our running example, the specifications of the London-dependent environment conditions PenalizedVehicleSIssuedFineOrChargePaid, PollutingVehiclesAdmitted, FineIssuedByOffenceType and PollutingByVehicleType are replaced by the following Brussels-dependent environment conditions, respectively:

$$
\begin{aligned}
& \text { Brussels : Penalized } \Leftrightarrow \text { FineIssued } \\
& \text { Brussels : Polluting } \Rightarrow \neg \text { Admitted } \\
& \text { Brussels }: \text { Polluting } \Leftrightarrow((\text { Diesel } \wedge \text { Euro } \leq 3) \vee(\text { Petrol } \wedge \text { Euro } \leq 1)) \\
& \text { Brussels }: \text { FineIssued } \Leftrightarrow((\text { FirstTime } \rightarrow \text { OneHundredFiftyFine }) \\
&\qquad(\neg \text { FirstTime } \rightarrow \text { TwoHundredFiftyFine }))
\end{aligned}
$$

whilst the London environment condition TwentyPaIDWhenCHARgePAid is deleted. The obvious anchor goal here is PollutingVenicleSPENALIZED since any LEZ enforcement system has to meet this.

\subsection{Checking Model Correctness}

This step takes a goal model $\widetilde{M}_{C^{\prime}}$-at start, this is the reference model $M_{C^{\prime}}^{0}$-and checks if all its AND-refinements are correct under the target context $C^{\prime}$ (steps $3 a$ and $3 b$ in Fig. 2). The outcome is a confirmation that either $(i)$ the input model is correct; ( $i i)$ the input model is complete and consistent but not minimal; or (iii) the input model is incomplete or inconsistent, and a counterexample trace.

Given an anchor goal $A G$ in $\mathcal{A}$, RESPIRE constructs satisfaction arguments for the completeness and consistency of its refinements first, and verifies these to generate counterexamples and witness examples if these arguments do not hold. An obvious option would be to construct these arguments between the anchor goal (as conclusion) and its "immediate" subgoals (in the premises) first, check the satisfaction of these, and learn an adaptation before recursively proceeding to the construction of arguments for descendent subgoals in a top-down fashion. This option would deem some computations wasteful as the satisfaction argument for a parent goal may become invalidated by adaptations to its subgoals. In contrast, RESPIRE constructs satisfaction arguments for refinement completeness/consistency between leaf goals and their ancestral anchor goals. This provides several benefits: $(i)$ the traces generated by the checks and used by the logic-based learning algorithm are guaranteed to be consistent with the agents' capabilities specification-this in turn guarantees that the adapted goals are realizable; (ii) the generated traces are more concrete and provide the learning algorithm with finer-grained examples highlighting which parts of the goal specifications need to be adapted; and (iii) unnecessary re-computations of adaptations to parents are avoided as changes to the leaf goals automatically produce changes to their parent goals, and ensure the progress of all ancestral refinements towards correct ones. The construction of satisfaction arguments for checking minimality is discussed in Section 6.1.3.

The three main checks RESPIRE conducts for verifying the adapted model's correctness are now further detailed.

6.1.1 Checking Completeness. RESPIRE first verifies the completeness of refinements of the anchor goal $A G$ in the input model under the target context $C^{\prime}$. In what follows, $\widetilde{M}_{C^{\prime}}^{i}$ denotes the goal model generated in the $i$ th iteration of the counterexample-guided learning loop, thus representing the $i$ th adaptation of the reference goal model $M_{C^{\prime}}^{0}$. Initially, $i=0$.

The verification is done by model checking that all leaf goals satisfy their anchor goal:

$$
\left\{\bigwedge \text { leaves }\left(A G, \widetilde{M}_{C^{\prime}}^{i}\right), P_{C^{\prime}}, E_{C^{\prime}}\right\} \mid=A G
$$

where leaves denotes the set of leaf goals descending from $A G$ in $\widetilde{M}_{C^{\prime}}^{i}$; a counterexample to the argument (5) is a witness to the incompleteness of $A G$ 's refinement.

In our example, the reference model $M_{\text {Brussels }}^{0}$ contains the anchor goal PollutingVeniclesPenalized specified as Polluting $\Rightarrow$ Penalized. Its refinement is incomplete since there is a trace in which the leaf goals FineSetByVehicleAndOffenceType and VehicleTypeCharged, specified as

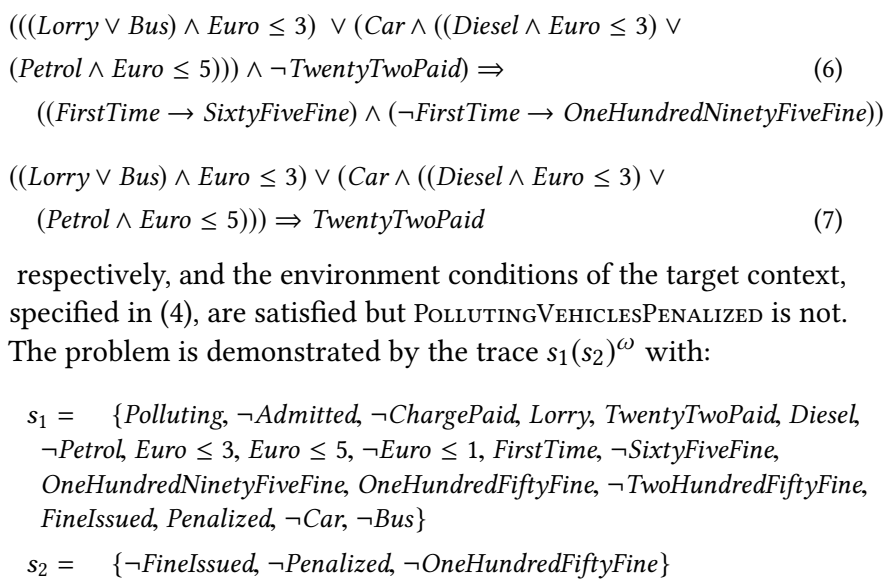

respectively, and the environment conditions of the target context, specified in (4), are satisfied but PollutingVehiclesPenalized is not. The problem is demonstrated by the trace $s_{1}\left(s_{2}\right)^{\omega}$ with:

The truth values for all variables are shown for the initial state, denoted $s_{1}$, followed by their values in consecutive states only if changed from the previous state. Otherwise, their truth values are assumed to persist. This counterexample violates PollutingVehiclesPenalized in $s_{2}$ where Polluting is true but Penalized is false.

If a counterexample to (5) is found, it is added to the set $\Sigma^{-}$ which accumulates all (negative) traces demonstrating incomplete refinements. This set is then used by the learner in the next step to prune out adaptations that falsify the anchor goals.

On the other hand, to guide the search towards correct adaptations, RESPIRE seeks to find (positive) traces that exhibit how anchor goals may be achieved in the target context. To this end, 
RESPIRE selects a leaf goal $L G$ in leaves $\left(A G, \widetilde{M}_{C^{\prime}}^{i}\right)$, at random or according to some ordering provided by the analyst. It then constructs and model-checks the argument

$$
\left\{\bigwedge \operatorname{anchors}\left(L G, \widetilde{M}_{C^{\prime}}^{i}\right), P_{C^{\prime}}, E_{C^{\prime}}\right\}=L G
$$

where anchors denotes the set of anchor goals that are ancestral to the leaf goal $L G$ in $\widetilde{M}_{C^{\prime}}^{i}$. Traces violating the above argument are called adaptation traces. They demonstrate situations in which the anchor goal and the pre-adapted leaf goal are not satisfied together under the target context (hence requiring an adaptation). In the LEZ example, we may consider the leaf goal FineSETByVEHICLEANDOFFENCETyPE whose payment of pollution charges is irrelevant in Brussels. (Relevance may be assessed from the target environment conditions; for instance, a goal is relevant if the propositional variables appearing in its goal specification also appear in at least one environment condition of the target context.) To generate an adaptation trace, RESPIRE checks the argument (8), where the specifications of FineSetByVehicLeAndOfFenceType as $L G$, and PollutingVehiclesPenalized as its single ancestral anchor, respectively. A counterexample to this is the trace $s_{1} s_{2}\left(s_{3}\right)^{\omega}$ :

$$
\begin{aligned}
s_{1}= & \{\text { Polluting, } \neg \text { Admitted, } \neg \text { ChargePaid, Lorry, Diesel, } \\
& \text { Euro } \leq 3, \text { TwentyTwoPaid, FirstTime, } \neg \text { SixtyFiveFine, } \\
& \neg \text { OneHundredNinetyFiveFine, OneHundredFiftyFine, } \\
& \text { FineIssued, Penalized, } \neg \text { TwoHundredFiftyFine }\} \\
s_{2}= & \{\neg \text { TwentyTwoPaid }\} \quad s_{3}=\{\text { TwentyTwoPaid }\}
\end{aligned}
$$

The original leaf goal is violated above as a lorry whose euro category is less than 3 and which did not pay the charge is not set the correct fine of SixtyFiveFine in $s_{1}$. This is acceptable behaviour in the target context where the correct fine of OneHundredFiftyFine for a first time offender is set. Note that as TwentyTwoPaid is irrelevant in the Brussels context, its truth value does not affect the acceptability of the trace since-we will see in Section 6.2-adaptations are restricted to the language relevant to the target context. Traces violating the argument (8) are added to the set $\Sigma^{+}$. A counterexample to the satisfaction of a leaf goal could be deemed unacceptable by the analyst. (Section 7 shows how our implementation of RESPIRE supports human-in-the-loop to assess the acceptability of and modify the counterexample before it is added to $\Sigma^{+}$.)

The output of a failed completeness check is two sets of traces: $\Sigma^{-}$, containing traces violating the anchor goal in the target context $C^{\prime}$, and $\Sigma^{+}$, containing traces that satisfy it in $C^{\prime}$.

6.1.2 Checking Consistency. To ensure that refinements in the adapted goal model are consistent, RESPIRE constructs and modelchecks the following argument.

$$
\left\{A G, P_{C^{\prime}}, E_{C^{\prime}}\right\} \models \neg \bigwedge \text { leaves }\left(A G, \widetilde{M}_{C^{\prime}}^{i}\right)
$$

A violation to (9) is a demonstration of the leaf goals' consistency. The satisfaction of this argument, on the other hand, shows that at least one of the goals in leaves $\left(A G, \widetilde{M}_{C^{\prime}}^{i}\right)$ cannot be satisfied together with the other leaf goals under the target context; thus requiring adaptation. In this case, RESPIRE selects a leaf goal $L G \in$ leaves $\left(A G, \widetilde{M}_{C^{\prime}}^{i}\right)$, searches for an adaptation trace violating $L G$ as per argument (8) and adds this trace to the set $\Sigma^{+}$.

Consistency checks are performed only once for each anchor goal. The reason is that the subsequent learning steps guarantee,

${ }^{3}$ The selection of leaf goals can be done at random automatically or user-driven. This is discussed in Section 7. by construction, that all adapted goals are true in each adaptation trace. Inconsistency can only arise between goals of the reference goal model $\widetilde{M}_{C^{\prime}}^{0}$ and the target environment conditions, leading to the vacuous satisfaction of the completeness property. The output of this check is an updated set $\Sigma^{+}$of adaptation traces.

6.1.3 Checking Minimality. If the AND-refinements of anchor goal $A G$ are complete and consistent, then RESPIRE proceeds to Step $3 b$ in Fig. 2, where every such refinement in $\widetilde{M}_{C^{\prime}}^{i}$ is checked for its satisfaction of the minimality property (3) of Section 2.1.

In contrast with completeness checks, minimality checks are conducted for every refinement starting from the anchor goal and its immediate subgoals, and recursively in a top-down fashion. Minimality checking considers the direct refinement of a parent goal, dropping a subgoal and checking for completeness of the remaining subgoals with respect to the parent goal. This top-down strategy avoids unnecessary checks for refinements that would be eliminated in Step 5 by the removal of ancestral refinements violating the minimality property. If a refinement is found to be not minimal, the procedure moves to Step 5 in Fig. 2.

\subsection{Learning Model Adaptations}

Step 4 in Fig. 2 takes as input the anchor goal $A G$, reference goal model $M_{C^{\prime}}^{0}$, target context $C^{\prime}$ and environment conditions $E_{C^{\prime}}$, agents' capabilities specifications $N$, and sets $\Sigma^{+}$and $\Sigma^{-}$produced by the completeness and consistency checks above. The aim of this step at the $i$ th iterated adaptation of the reference model is to find a model adaptation $\widetilde{M}_{C^{\prime}}^{i+1}$ where:

(a) for every $\sigma \in \Sigma^{+}, \widetilde{G} \in \widetilde{M}_{C^{\prime}}^{i+1}$, we have $\sigma \mid \widetilde{G}$;

(b) for every $\sigma \in \Sigma^{-}$, we have $\sigma \vDash \wedge$ leaves $\left(A G, \widetilde{M}_{C^{\prime}}^{i+1}\right)$;

(c) each anchor goal $A G \in \mathcal{A}$ is unchanged in $\widetilde{M}_{C^{\prime}}^{i+1}$;

(d) every leaf goal $\widetilde{G}$ in $\widetilde{M}_{C^{\prime}}^{i+1}$ is realizable;

(e) $\widetilde{M}_{C^{\prime}}^{i+1}$ is a maximally local adaptation of $M_{C^{\prime}}^{0}$;

(f) $\widetilde{M}_{C^{\prime}}^{i+1}$ is maximally similar to $M_{C^{\prime}}^{0}$.

The second requirement above ensures the correctness of the adapted model; since every trace in $\Sigma^{-}$violates an anchor goal, it should also violate at least one goal in its refinement.

This step deals with a restricted form of the adaptation task defined in Section 4; it considers correctness of the adapted model w.r.t. traces in $\Sigma^{-}$and $\Sigma^{+}$. We call this a trace-driven adaptation task. We thus introduce a weaker notion of completeness and consistency. Given a trace $\sigma$, parent goal $P G$ and subgoals $\left\{S G_{i}\right\} \subseteq P G_{\text {RefinedBy }}$, $\left\{S G_{i}\right\}$ is a trace-complete refinement w.r.t. $\sigma$, iff

$$
\sigma \mid=\left\{S G_{1}, \ldots, S G_{n}, E\right\} \text { implies } \sigma \mid=P G
$$

It is said to be a trace-consistent refinement w.r.t. $\sigma$ iff

$$
\sigma \mid=\left\{S G_{1}, \ldots, S G_{n}, E\right\}
$$

A goal model is trace-consistent and -complete w.r.t. $\sigma$ if every refinement is trace-consistent and -complete w.r.t. $\sigma$, respectively.

To compute such adaptations, the learning step comprises four main sub-steps: $(i)$ encode the trace-driven adaptation task into a learning-based revision task; (ii) define the space of preferred adaptations; (iii) compute an adaptation as a solution to the revision task; and (iv) encode the solution to the revision task back into an adapted goal model. The first three sub-steps are described below; the last is simply the inverse of the first. 
6.2.1 Encoding the adaptation problem. Our learning algorithm relies on a canonical representation of goal models, on the semantics of correct refinements, and on the syntax and semantics of the class of MLTL formulas expressible as normal logic programs. We thus develop an encoding $(\llbracket \rrbracket)$ that extends the one presented in [8] with the rules below. The symbol $l_{\phi}$ (resp. $l_{\sigma}$ ) corresponds to a constant uniquely representing the formula $\phi$ (resp. trace $\sigma$ ); $l_{\alpha}$ is a constant representing agent $\alpha$ 's name. $\operatorname{Mon}_{\alpha} \subseteq \mathcal{P}$ (resp. $\operatorname{Ctrl}_{\alpha} \subseteq \mathcal{P}$ ) denotes the set of monitorable (resp. controllable) propositional variables for this agent. The main property of this encoding is that, for any given trace $\sigma$ and MLTL safety property $\phi$, we have $\sigma, i=\phi$ iff $\operatorname{true}\left(l_{\phi}, i, l_{\sigma}\right) \subset \mathbb{M}(\llbracket \phi \rrbracket \wedge \llbracket \sigma \rrbracket)$.

$$
\begin{aligned}
& \llbracket G_{\text {Resp }} \rrbracket \stackrel{\text { def }}{=} \text { assigned_to }\left(G_{\text {Name }}, G_{\text {Resp }}\right) \\
& \llbracket G_{\text {Anchor }} \rrbracket \stackrel{\text { def }}{=} \text { anchor }\left(G_{\text {Name }}\right) \quad \text { if } G_{\text {Anchor }}=\text { true else empty } \\
& \llbracket C \rrbracket \stackrel{\text { def }}{=} \text { context }\left(P_{C}\right) \\
& \llbracket N \rrbracket \stackrel{\text { def }}{=} \bigwedge\left\{\text { monitorable }\left(l_{\alpha}, x\right) \mid x \in \text { Mon }_{\alpha} \text { and } \alpha \in N\right\} \wedge \\
& \wedge\left\{\text { controllable }\left(l_{\alpha}, x\right) \mid x \in \text { Ctrl }_{\alpha} \text { and } \alpha \in N\right\} \\
& \llbracket \Sigma \rrbracket \stackrel{\text { def }}{=} \bigwedge\left\{\text { true }\left(x, i, l_{\sigma}\right) \mid x \in \sigma(i) \text { and } \sigma \in \Sigma\right\} \wedge \\
& \bigwedge\left\{\text { false }\left(x, i, l_{\sigma}\right) \mid x \notin \sigma(i) \text { and } \sigma \in \Sigma\right\}
\end{aligned}
$$

$\llbracket G \rrbracket$ encodes $G$ 's definition. The background theory $B$ comprises the encoding of anchor goals' definitions in $M_{C^{\prime}}^{0}$ (excluding their refinement links), the target context and its environment conditions, agent capabilities' specification and traces in $\Sigma^{+}$and $\Sigma^{-}$:

$$
\begin{aligned}
\wedge\left\{\llbracket A G \backslash \text { RefinedBy } \rrbracket \mid A G \in \operatorname{anchors}\left(L G, M_{C^{\prime}}^{0}\right)\right\} \wedge \\
\wedge \llbracket C^{\prime} \rrbracket \wedge \llbracket E_{C^{\prime}} \rrbracket \wedge \llbracket N \rrbracket \wedge \llbracket \Sigma^{+} \rrbracket \wedge \llbracket \Sigma^{-} \rrbracket
\end{aligned}
$$

In addition, $B$ also comprises a declarative representation of the semantics of trace-complete refinements, for all $P G \in M_{C^{\prime}}^{0}$ :

$$
\begin{aligned}
& \bigwedge\left\{\operatorname{true}\left(S G_{\text {Name }}, l_{\Sigma}\right) \mid S G \in M_{C^{\prime}}^{0} \text { and } S G \in P G_{\text {RefinedBy }}\right\} \\
& \rightarrow \operatorname{true}\left(P G_{\text {Name }}, l_{\Sigma}\right. \\
& \text { false }\left(P G_{\text {Name }}, l_{\Sigma}\right) \rightarrow \\
& \neg \wedge\left\{\operatorname{true}\left(S G_{\text {Name }}, l_{\Sigma}\right) \mid S G \in M_{C^{\prime}}^{0} \text { and } S G \in P G_{\text {RefinedBy }}\right\}
\end{aligned}
$$

where $l_{\Sigma}$ is a universally quantified variable over $\Sigma^{+} \cup \Sigma^{-}$. The first is a direct encoding of (10). The latter encodes the requirement (b) described above. The trace-consistent refinement property (11) is encoded implicitly in the examples as discussed below. To learn model adaptations whose refinements comprise new goals, $B$ may be extended with placeholders for goal definitions.

The revisable theory $K$, instead, includes an encoding of definitions of all goals descending from an anchor goal that is an ancestor to the selected leaf goal, and an encoding of the refinement relations between anchor goals and their subgoals as follows.

$$
\begin{aligned}
& \wedge\left\{\llbracket A G_{\text {RefinedBy}_{G}} \rrbracket \mid A G \in \operatorname{anchors}\left(L G, M_{C^{\prime}}^{0}\right)\right\} \wedge \\
& \wedge\left\{\llbracket G \rrbracket \mid G \in \operatorname{desc}\left(A G, M_{C^{\prime}}^{0}\right) \text { and } A G \in \operatorname{anchors}\left(L G, M_{C^{\prime}}^{0}\right)\right\}
\end{aligned}
$$

The examples $\mathrm{O}^{+} \cup \mathrm{O}^{-}$capture the trace-consistent refinements' requirement-it necessitates all adapted goal specifications in $\widetilde{K}$ to hold in every trace in $\Sigma^{+}$and not in $\Sigma^{-}$:

$O^{+}=\left\{\operatorname{true}\left(G_{\text {Name }}, l_{\sigma}\right) \mid G \in M_{C^{\prime}}^{0}\right.$ and $\left.\sigma \in \Sigma^{+}\right\}$and

$O^{-}=\left\{\right.$false $\left(A G_{\text {Name }}, l_{\sigma}\right) \mid A G \in M_{C^{\prime}}^{0}$ and $\left.\sigma \in \Sigma^{-}\right\}$.

To guide the learning procedure towards adaptations yielding correct refinements and realizable leaf goals, RESPIRE specifies: $(i)$ a mode bias $I$ that restricts the propositional variables allowed to appear in goal specifications to those "relevant" to the new context; and (ii) structural constraints $D$ over the set of specifications and refinements relations to which any candidate goal model must adhere. The interested reader is referred to the extended version of this paper in [43] for details of the mode bias $I$ and structural constraints $D$. Note that the encoding for all the above definitions is generated once for every selected anchor goal. Only new counterexamples and adaptation traces are encoded in subsequent iterations.

6.2.2 Defining preferred adaptations. One key novelty in our learning procedure is the use of soft preferences to guide the revision task towards adaptations that meet the similarity and locality requirements defined in Section 4. RESPIRE implements these notions by defining an adaptation cost and adaptation impact.

Adaptation cost. When computing adaptations, RESPIRE applies a series of change operations to goal definitions encoded in $K$. These include the following operations: modify a type, add/delete a refinement relation, add/delete a literal to/from a specification or delete a responsibility assignment. The application of these operations defines an adaptation mapping between goal definitions. Given the definition of two goals $G 1$ and $G 2$, an adaptation mapping is the smallest set of change operations that can be applied to $G 1$ to obtain G2. The cost of a goal adaptation is given by the size of the adaptation mapping between $G 1$ and $G 2$. We assume a uniform cost of one for change operations. The total cost of an adaptation of $M$ is the sum of all goal adaptations' costs. The learning aims at finding adaptations that minimize this cost.

The adaptation cost implicitly captures a measure of similarity between two sets $\gamma_{1}$ and $\gamma_{2}$ based on Tversky's [47]:

$$
\begin{aligned}
& \operatorname{similar}\left(\gamma_{1}, \gamma_{2}\right)=\left|\left\{x \mid x \in \gamma_{1} \cap \gamma_{2}\right\}\right|- \\
& \quad \mid\left\{x \mid x \in \gamma_{1} \text { and } x \notin \gamma_{2}\right\}|-|\left\{x \mid x \notin \gamma_{1} \text { and } x \in \gamma_{2}\right\} \mid
\end{aligned}
$$

(When $\gamma_{1}, \gamma_{2}$ are elements, then $\operatorname{similar}\left(\gamma_{1}, \gamma_{2}\right)=1$ if $\gamma_{1}=\gamma_{2}$; 0 otherwise.) Similarity between goals is thereby defined as the similarity between their types, specification, refinements and responsibility assignments: $\operatorname{similar}(G, \widetilde{G})=\operatorname{similar}\left(G_{\text {Type }}, \widetilde{G}_{\text {Type }}\right)+$ $\operatorname{similar}\left(G_{\text {Spec }}, \widetilde{G}_{\text {Spec }}\right)+\operatorname{similar}\left(G_{\text {RefinedBy }}, \widetilde{G}_{\text {RefinedBy }}\right)+\operatorname{similar}($ $\left.G_{\text {Resp }}, \widetilde{G}_{\text {Resp }}\right)$, where $\operatorname{similar}\left(G_{\text {Spec }}, \widetilde{G}_{\text {Spec }}\right)$ is the sum of the similarity measures between their respective Current- and Target-condition and temporal operators. ${ }^{4}$ For similar $\left(\right.$ Current $_{G}$, Current $\left.\widetilde{G}_{\widetilde{G}}\right)$, for instance, the first term of Equation (12) corresponds to literals that are unchanged in the Current-condition of $G$ in $\widetilde{K}$. The second corresponds to those deleted from the Current-condition of $G$ in $K$ and the third corresponds to those added to it. (Goals not included in any refinement link in $\widetilde{M}$ are considered to be deleted from $M$.)

The similarity measure between goal models is defined as the sum of the similarity measures between every goal $G$ in $M$ and its adaptation $\widetilde{G}$ in $\widetilde{M}$. The cost of the adaptation, denoted $\operatorname{cost}(M, \widetilde{M 1})$, is the inverse of the similarity measure. We say that an adaptation $M 1$ of $M$ is less costly than $\widetilde{M 2}$ iff $\operatorname{similar}(M, \widetilde{M 1})>\operatorname{similar}(M, \widetilde{M 2})$. The learning procedure aims to minimize the adaptation cost (i.e., maximize the similarity between a goal model and its adaptation). Adaptation impact. Impact measures how local the changes are in the reference goal model $M_{C^{\prime}}^{0}$. To measure the impact of an adaptation, we define a notion of distance between goals in a goal model. The distance between two connected goals $G 1$ and $G 2$ in

${ }^{4}$ Note that we consider here syntactic similarity. We reserve discussion on semantic similarity for Section 9. 
model $M$, denoted by $\operatorname{dist}(G 1, G 2, M)$, is the minimum number of refinement links connecting them. RESPIRE computes this measure by keeping track of all leaf goals selected in Step $3 a$. We denote this set as selected $(M)$. The locality of an adaptation $\widetilde{M}$ to $M$ is calculated by summing the distances between every leaf goal $L G \in$ selected $(M)$ and goal $X$ in $M$ where both have been modified in $\widetilde{M}$ and, in the case where a new goal $X$ is added to $\widetilde{M}$, the distances between the selected $L G$ and between the goal $X$ and some common ancestral anchor appearing in both $M$ and $\widetilde{M}$ as given below.

$$
\begin{gathered}
\operatorname{local}(M, \widetilde{M}, L G)=\sum_{x \in \mathcal{I}_{1}(L G)} x+\sum_{y \in \mathcal{I}_{2}(L G)} y+\sum_{z \in \mathcal{I}_{3}(L G)} z \\
\mathcal{I}_{1}(L G) \stackrel{\text { def }}{=}\{\operatorname{dist}(L G, X, M) \mid X \in M \text { and } \widetilde{X} \in \widetilde{M} \text { and } \\
\left.X_{\text {Name }}=\widetilde{X}_{\text {Name }} \text { and } X \not \equiv \widetilde{X}\right\} \\
\mathcal{I}_{2}(L G) \stackrel{\text { def }}{=}\{\operatorname{dist}(L G, X, M) \mid X \in M \text { and } X \notin \widetilde{M}\} \\
\mathcal{I}_{3}(L G) \stackrel{\text { def }}{=}\left\{\operatorname{dist}\left(L G, G^{*}, M\right)+\operatorname{dist}\left(G^{*}, X, \widetilde{M}\right) \mid G^{*} \in M \cap \widetilde{M}\right. \\
\text { and } G^{*} \text { is the closest ancestor to } L G \text { in } M \\
\left.G^{*} \in \text { ances }(X, \widetilde{M}) \text { and } X \notin M \text { and } X \in \widetilde{M}\right\}
\end{gathered}
$$

The impact of an adaptation $\widetilde{M}$ of $M$, denoted $\operatorname{impact}(M, \widetilde{M})$, is the sum of the locality measures w.r.t. all $L G \in \operatorname{selected}(M)$. Thus an adaptation $\widetilde{M}_{1}$ has less impact on (i.e., more local in) $M$ than an adaptation $\widetilde{M}_{2}$ iff $\operatorname{impact}\left(M, \widetilde{M}_{1}\right)<\operatorname{impact}\left(M, \widetilde{M}_{2}\right)$. The learning procedure aims to minimize the impact of an adaptation.

6.2.3 Computing adaptations. The learning algorithm attempts to find an inductive solution that optimizes the preference criteria described above. Conflicts may arise when attempting to minimize both the adaptation cost and impact. To resolve these, we deploy a pre-emptive optimization strategy in which cost minimization is prioritized over impact minimization. If a solution exists, the adapted goal specifications are guaranteed to hold in all traces of $\Sigma^{+}$but in none of $\Sigma^{-}$-thus meeting the trace-consistent and -complete refinement properties. For our LEZ example, the learning step returns for instance the following adaptation of FinESETBYVEHICLEANDOFFENCETyPE, compactly specified as:

$$
\begin{aligned}
(\text { Euro } \leq & 3 \vee(\text { Diesel } \wedge \text { Euro } \leq 3) \vee \text { Petrol }) \Rightarrow \\
& ((\text { FirstTime } \rightarrow \text { OneHundredFiftyFine }) \wedge \\
& (\neg \text { FirstTime } \rightarrow \text { TwoHundredFiftyFine }))
\end{aligned}
$$

Since the leaf goals (6) and (7), both of which appear in $\widetilde{M}$, were modified, we have $\operatorname{impact}\left(M, \widetilde{M}_{\text {Brussels }}^{1}\right)=4$ as there are 4 refinement links connecting them (i.e., $\mathcal{I}_{1}(L G)=4$ and $\mathcal{I}_{2}(L G)=\mathcal{I}_{3}(L G)=0$ ). $\operatorname{cost}\left(M, \widetilde{M}_{\text {Brussels }}^{1}\right)=14$ for the deletion of the literals Lorry, Bus, Car, TwentyTwoPaid and Euro $\leq 5$ (which appear in both), and SixtyFiveFine and OneHundredNinetyFiveFine from (6) and and the addition of OneHundredFiftyFine and TwoHundredFiftyFine to (6) Once a solution is generated, the adapted model $\widetilde{M}_{C^{\prime}}^{i+1}$ is checked for complete refinements w.r.t. $A G$ as per Section 6.1.1.

\subsection{Updating adaptations towards minimality}

As noted in Section 6.1.3, once all refinements of an anchor goal in the adapted goal model are complete and consistent, RESPIRE conducts a minimality check for every parent goal in the adapted model (Step $3 b$ in Fig. 2). The aim of this step is to remove subgoals that violate the minimality property of (3). Starting at the anchor goal, if removing a direct subgoal maintains completeness, the subgoal and its descendants are removed. Finding a minimal set of subgoals satisfying (3) is NP-hard. However, it is assumed that parent goals in well-structured models have a small number of immediate children [48] making this computation tractable.

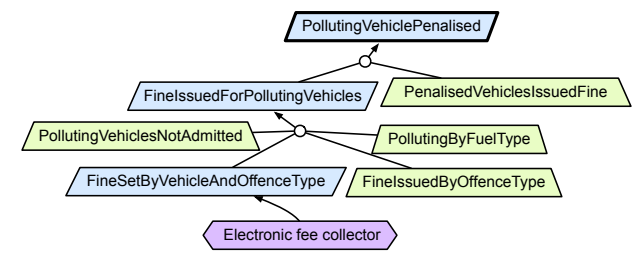

Figure 3: Adapted goal model for Brussels LEZ.

In our LEZ example, two more iterations over the anchor goal PollutingVehiclesPenalized result in a complete refinement. The minimality check finds that dropping the subgoal CHARgePAidWHENPollutingVehicleAdmitted maintains completeness of the goal PolLutingVehiclesPenalized's refinement. Therefore, this subgoal and its subtree are removed. The updated goal model is shown in Fig. 3.

\section{IMPLEMENTATION}

We developed a prototype toolset (in Python) implementing the adaptation procedure detailed in Section 6. The toolset integrates a number of third-party libraries and software. We use the Python Boolean library [1] to rewrite the Current-conditions of specifications into a standard form. We use the NuSMV2 2.6 symbolic model checker [16] to ( $i$ ) check the correctness of goal models; and ( $i i)$ generate counterexamples. We built upon the RASPAL learning-based revision tool [10] for our learning engine. We built an automated translator to convert goal specifications into NuSMV and RASPAL syntax, and vice versa.

The tool can run in fully automated mode or in interactive mode. The automated mode forms the base setting in which: $(i)$ all propositional variables appearing in the environment conditions of the target context, agents' capabilities specifications and anchor goals are considered when computing adaptation; (ii) leaf goals of the anchor goal are selected at random when verifying argument (8); (iii) all generated adaptation traces are accepted (without modification); and finally $(i v)$ the size of the adapted goal model is fixed.

The interactive mode supports various degrees of human control including: (i) selecting the variables that may appear in the target model; (ii) selecting the leaf goal to be checked; (iii) confirming the suitability of the automatically generated adaptation trace; (iv) providing an alternative adaptation trace if not; and finally $(v)$ enlarging the size of the search space by allowing RESPIRE to increase the number of goals in the target model or its refinement depth, or to explore alternative refinement tactics. Each of these features can be enabled when RESPIRE is launched. They are introduced to speed up the adaptation process towards relevant models.

\section{EVALUATION}

In this section, we evaluate our approach in terms of its correctness and applicability to real-world problems.

\subsection{Correctness}

Theorem 1 (TERmination). The RESPIRE adaptation procedure is guaranteed to terminate.

Proof sketch. There are three loops in the adaptation procedure. The loop over anchor goals terminates as the set of anchor goals is 
finite. The counterexample-guided learning loop within the dashed lines in Fig. 2 is guaranteed to terminate since: $(i)$ counterexamples and witness examples are accumulated in the sets $\Sigma^{+}$and $\Sigma^{-}$thus ensuring that a new goal model is learned in every iteration that is semantically different from previous ones; and (ii) the adaptation space is finite since the sets of change operations and of propositional variables are finite. The loop for minimality checking and model updating also terminates as the set of subgoals is finite.

Theorem 2 (SoundNess AND COMPLETENESS). Let $\Sigma^{+}$and $\Sigma^{-}$ be sets of traces generated by RESPIRE for the adaptation problem $\left\langle E, C, M, C^{\prime}, \mathcal{A}, N\right\rangle$. If RESPIRE returns a goal model $\widetilde{M}_{C^{\prime}}$, then $\widetilde{M}_{C^{\prime}}$ is correct under the target context. If RESPIRE returns $\perp$, then no adaptation exists in the constrained search space, that is trace-consistent and-complete w.r.t. $\Sigma^{+}$and $\Sigma^{-}$under $C^{\prime}$.

Proof sketch. Soundness is guaranteed since RESPIRE only constructs well-formed goal models that satisfy the correctness properties (1-3) of Section 2. Moreover, the learning procedure fails in two cases: $(i)$ the sets of examples provided by the analyst are not well-separated (i.e., a trace can appear as both a positive and negative); or ( $i i)$ no solution exists within the search space.

Theorem 3 (Similarity AND locality). Let $\left\langle E, C, M, C^{\prime}, \mathcal{A}, N\right\rangle$ be an adaptation problem, $\Sigma^{+}$and $\Sigma^{-}$the traces generated by RESPIRE and $\widetilde{M}_{C^{\prime}}$ an adaptation solution. Then $\widetilde{M}_{C^{\prime}}$ is a maximally similar adaptation to $M_{C}^{0}$. It is a maximally local adaptation, within the space of a maximally similar adaptations.

This follows from the definition of the reward function in the learning step (see Section 6.2). RESPIRE supports the search for similar goal models by providing the learning step with the reference goal model $M_{C^{\prime}}^{0}$ as input. As the search for a solution is driven by the sample of traces used, a local "minimal" solution to the adaptation problem, rather than a global minimal one is ensured.

\subsection{Applicability}

We briefly report on the application of RESPIRE to two real computeraided ambulance dispatch (CAD) systems: one for Belgium [42] and one for Wales [45]. Three common anchor goals were considered, yielding three sub-models to be adapted from Belgium to Wales (B-W) and three vice versa (W-B). A description of the number of goals and environment conditions in the sub-models rooted on the same anchor goal across the two contexts is given in Table 1.

\begin{tabular}{|c|c|c|c|c|}
\hline Anchor ID & $\#_{B}$ & ${ }^{\mathrm{G}_{B}}$ & ${ }^{\mathrm{E}} \mathrm{E}_{W}$ & $\#_{\mathrm{G}_{W}}$ \\
\hline 1 & 4 & 2 & 2 & 3 \\
2 & 14 & 4 & 7 & 3 \\
3 & 3 & 2 & 5 & 4 \\
\hline
\end{tabular}

Table 1: Description of source/target sub-models considered.

Our RESPIRE tool was applied in two modes: the fully automated mode and the interactive mode. In the interactive mode, the analyst could: ( $i$ ) assess and manually change the automatically generated adaptation traces to suit the target context; and (ii) increase the the size of any adapted model to be computed. The first author acted as the analyst. All other steps were done automatically. Experiments were conducted using a $2.8 \mathrm{GHz}$, Intel Core i7 processor.

The fully automated mode often terminated unsuccessfully in at most two iterations owing to either the automatically generated adaptation traces being inadequate for the target context, the need to increase size of the adapted model or missing environment properties. Consider the anchor goal "an ambulance shall arrive at the scene of a reported incident on time". Checking its completeness in the Wales context results in an adaptation trace for the leaf goal MaxinterventionTimeIs12Minutes where an ambulance requires more than 14 minutes to arrive (without any problem along the way): this scenario is inadequate in the Wales context. By modifying the ambulance's arrival time in the trace, as indicated by $E_{W}$, RESPIRE finds the correct solution. Hence, we focus in our discussion on the interactive mode experiments.

RESPIRE terminated successfully for all six adaptation problems. It effectively covered different types of adaptations requiring modifications to: goal specifications (GS); refinement links (RL); refinement tactics (RT) by transforming a case-driven goal refinement into a milestone-driven one [48] (and vice versa); and the goal model's depth (RD). Table 2 gives a summary of the results.

\begin{tabular}{|c|c|c|c|c|c|c|}
\hline & \# $\operatorname{Lrn~G}{ }_{T}$ & \# Min. $\mathrm{G}_{T}$ & \#Iter. & Type & Avg. cost & Avg. reward \\
\hline B-W-1 & 2 & 2 & 2 & GS, RL & 4 & 1 \\
\hline B-W-2 & 3 & 2 & 2 & GS, RT & 12 & 2 \\
\hline B-W-3 & 3 & 3 & 1 & GS, RD, RL & 14 & 4 \\
\hline W-B-1 & 2 & 1 & 1 & GS & 10 & 2 \\
\hline W-B-2 & 3 & 3 & 6 & GS, RT, RL & 12.5 & 3 \\
\hline W-B-3 & 3 & 1 & 1 & GS, RD & 14 & 3 \\
\hline
\end{tabular}

Table 2: Summary of second experiment results.

\#Lrn. $\mathrm{G}_{T}$ gives the number of learned goals in the adapted submodel of the target context. \#Min. $\mathrm{G}_{T}$ is the number of goals in the updated goal model after the minimality check and model updating. \#lter. is the number of completeness checks performed until RESPIRE terminates. The column Type gives the form of adaptation involved. The last two columns give the average costs and impact of all adapted models computed until RESPIRE terminates.

For instance, we know from Table 1 that the source sub-model rooted on the anchor goal with ID 1 contained four environment conditions which were replaced by two environment conditions for the Wales context. The original Belgium sub-model contained two goals (including the anchor goal), whilst the Wales one contained three. From Table 2, we see that RESPIRE learned two goals in solving the adaptation problem B-W for the sub-models rooted on the anchor goal with ID 1 (i.e., B-W-1); both were maintained after the minimality check. Two iterations were needed to termination. The adaptation involved changes to the goal specification and addition of a refinement link (of distance 2). The average cost of the adapted models computed over the iterations is 4 and the average impact is 1. We notice that $\mathrm{RD}$ adaptations are typically more costly as they require learning the full specification of the new leaf goals.

Fig. 4 plots the time in seconds (y-axis) spent by the model checking (blue) and the learning (red) components in an application of RESPIRE to each adaptation problem ( $\mathrm{x}$ - axis). The variance in each box is over time spent in iterations of the counterexampleguided learning loop. Overall, the time spent by the two components did not exceed 1.6s and the time spent by each component increased linearly as the number of iterations increased.

\section{DISCUSSION}

Scalability. RESPIRE's scalability relies on that of the model checking and learning procedures. We use NuSMV, an efficient BDDbased checker heavily deployed in practice. The learning engine is 


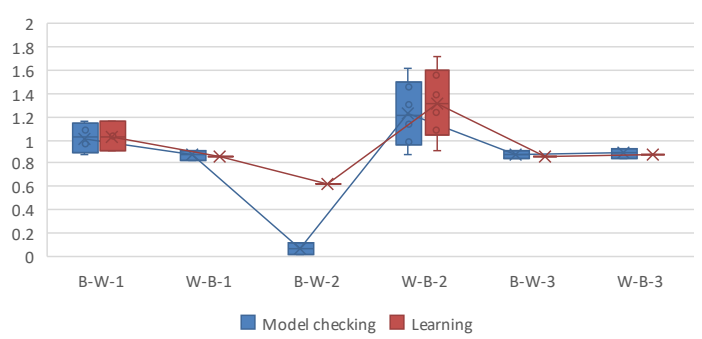

Figure 4: Performance of RESPIRE in interactive mode.

implemented using the answer set solver Clingo [23] whose scalability is comparable to SAT solvers. A crucial point is the size of the search space. The interactive mode, therefore, also allows the analyst to restrict the search space to a practical scope.

Human control. Having analysts in the loop is important in producing adequate models for unseen contexts. This was particularly apparent in the choice of adaptation traces as these guide the outcome of the learning procedure and subsequently generated counterexamples. We argue that RESPIRE's benefits are not hindered by this; modifying trace examples is often considered less error-prone than modifying the specifications [25]. In our experience, trace modification typically involved changing the truth values of one or two propositional variables in the automatically produced trace at most. We also found that allowing the analyst to assess the adaptation traces often highlighted implicit environment conditions, e.g., Critical $\Rightarrow \neg$ HighlyCritical in W-B-2. Though the fully automated mode terminated unsuccessfully initially for W-B-2, two goals were correctly computed. Increasing the number of goals in the adapted model in interactive mode, here by 1 , lead to successful termination.

Semantic similarity. RESPIRE searches for syntactically similar goal models which may yield semantically different models (i.e., in terms of the set of behaviour they prescribe). We can leverage for this by extending the step $3 a$ with an additional query to the model checker, namely, $\wedge \operatorname{desc}\left(A G, M_{C^{\prime}}^{0}\right) \wedge P_{C^{\prime}} \wedge E_{C^{\prime}}=\neg A G$. Counterexamples are then added to $\Sigma^{+}$. We will treat this in future work.

\section{RELATED WORK}

Much of RE work on adaptation so far has focused on reconfiguration within a fixed, predefined adaptation space [3, 4, 9, 28, 31, 34] None is driven by satisfaction arguments. In [31], a general problem definition framework is proposed for requirements-based runtime adaptations as an alternative to [49] which this work builds on The work in [4] also presents a notion of contextual goal models where goals and refinements are decorated with context conditions that are monitored at run-time for switching to more appropriate alternative refinements. The work in [24] addresses the selection of most appropriate configurations for a dynamic software product line. The requirements there are assumed to be known at RE time and are fixed. Our technique might be seen as complementary by learning modified requirements that could be taken as input.

The techniques proposed in [20] and [39] compute new specifications that satisfy modified requirements. In both techniques, the computed specifications are evaluated in terms of: $(a)$ the number of elements in common with the current specifications; $(b)$ the change effort required to modify the current specifications; and (c) the reuse of previous specifications. Our technique might complement these approaches by providing the modified requirements as input.

Learning-based solutions include [8, 46]. In [46], the authors define a probabilistic logic-based learning approach to revise behaviour models for reactive planning at run-time. Their focus is restricted to revising probabilities over environment assumptions (expressed as condition-event invariants). The work of [8], as noted in Section 1, differs substantially, including in: (a) purpose and problem addressed (finding countermeasures to obstacles in a single environment vs. finding model adaptations across varying environments); (b) method (type of model-checking and learning tasks, when/how to perform these); (c) degrees of automation; $(d)$ types and properties of model revisions (e.g., refinement minimality); and (e) specifications' semantics (event-based vs. state-based-the latter being better suited for reasoning about goal models [48]). As in [8], however, goal models are represented as normal logic programs and our inductive learning solutions can be classified as oracle-guided.

RESPIRE shares its essence with counterexample-guided learning methodologies, e.g., for invariant discovery [21]. The closest work to our learning methodology are [6, 7]. Unlike ours, however, $(a)$ their learning approaches are based on incremental refinement, which reduces the prescribed behaviour monotonically; $(b)$ preferences are not specified over the solution space; and (c) both approaches assume an event-driven asynchronous semantics of goal models.

\section{CONCLUSION AND FUTURE WORK}

The adequacy, completeness and consistency of software requirements depend on the software environment. Environment conditions are involved in satisfaction arguments, an important aspect of the RE process [49]. In varying environments, such arguments may be invalidated. A tool-supported approach was presented for adapting a given goal model automatically to new environment conditions. This approach combines verification-learning cycles constrained by preserving higher-level goals and by minimizing the adaptation cost and impact. Unlike other approaches to requirements adaptation, the space of possible changes needs not be explicit in advance. The application of RESPIRE to documented variations of a real ambulance system revealed that our approach goes far beyond mere renaming of variables in goal specifications.

This work builds a theoretical foundation for and assesses the feasibility of learning requirements adaptations. Further experiments into factors affecting the performance of RESPIRE, such as leaf goal selection and its usability is part of our future work. Common challenges of using formal methods at RE time remains. In particular, the environment knowledge used in satisfaction arguments has to be made explicit; complementary means are thus needed for handling the common tacit knowledge problem in RE. In addition, we intend to expand RESPIRE for runtime adaptations, e.g., using a runtime verifier. 


\section{REFERENCES}

[1] [n. d.]. ([n. d.]). http://formal.cs.utah.edu:8080/pbl/PBL.php.

[2] European Environment Agency. 2019. Low Emission Zones. (2019). http: //urbanaccessregulations.eu/low-emission-zones-main/.

[3] Germán H Alférez and Vicente Pelechano. 2012. Dynamic Evolution of ContextAware Systems with Models at Runtime.. In MoDELS. Springer, 70-86.

[4] Raian Ali, Fabiano Dalpiaz, and Paolo Giorgini. 2010. A goal-based framework for contextual requirements modeling and analysis. Requirements Engineering 15, 4 (2010), 439-458.

[5] Raian Ali, Fabiano Dalpiaz, Paolo Giorgini, and Vítor E. Silva Souza. 2011. Requirements Evolution: From Assumptions to Reality. In Proceedings of the 12th International Conference Enterprise, Business-Process and Information Systems Modeling. 372-382.

[6] Dalal Alrajeh, Jeff Kramer, Alessandra Russo, and Sebastián Uchitel. 2009. Learning operational requirements from goal models. In Proceedings of the 31st International Conference on Software Engineering, ICSE 2009, May 16-24, 2009, Vancouver Canada, Proceedings. 265-275.

[7] Dalal Alrajeh, Jeff Kramer, Axel Van Lamsweerde, Alessandra Russo, and Sebastián Uchitel. 2012. Generating obstacle conditions for requirements completeness. In Software Engineering (ICSE), 2012 34th International Conference on. IEEE, 705-715.

[8] Dalal Alrajeh, Axel van Lamsweerde, Jeff Kramer, Alessandra Russo, and Sebastián Uchitel. 2016. Risk-driven revision of requirements models. In Proceedings of the 38th International Conference on Software Engineering. 855-865.

[9] Konstantinos Angelopoulos, Vítor E. Silva Souza, and John Mylopoulos. 2015 Capturing Variability in Adaptation Spaces: A Three-Peaks Approach. In Proceedings of the 34th International Conference Conceptual Modeling. 384-398.

[10] Duangtida Athakravi, Dalal Alrajeh, Krysia Broda, Alessandra Russo, and Ken Satoh. 2014. Inductive Learning Using Constraint-Driven Bias. In Inductive Logic Programming - 24th International Conference, ILP 2014, Nancy, France, September 14-16, 2014, Revised Selected Papers. 16-32.

[11] Duangtida Athakravi, Domenico Corapi, Krysia Broda, and Alessandra Russo. 2013. Learning Through Hypothesis Refinement Using Answer Set Programming In Inductive Logic Programming - 23rd International Conference (ILP). 31-46.

[12] Armin Biere, Alessandro Cimatti, Edmund Clarke, and Yunshan Zhu. 1999. Symbolic Model Checking without BDDs. In Tools and Algorithms for the Construction and Analysis of Systems, W. Rance Cleaveland (Ed.). Springer Berlin Heidelberg, 193-207.

[13] Jim Buckley, Tom Mens, Matthias Zenger, Awais Rashid, and Günter Kniesel 2005. Towards a Taxonomy of Software Change. Journal of Software Maintenance and Evolution: Research and Practice 17, 5 (2005), 309-332.

[14] Antoine Cailliau and Axel Van Lamsweerde. 2019. Runtime Monitoring and Resolution of Probabilistic Obstacles to System Goals. ACM Trans. Auton. Adapt. Syst. 14, 1 (2019), 3:1-3:40.

[15] Antoine Cailliau and Axel van Lamsweerde. 2014. Integrating exception handling in goal models. In Requirements Engineering Conference (RE), 2014 IEEE 22nd International. IEEE, 43-52.

[16] Alessandro Cimatti, Edmund M. Clarke, Enrico Giunchiglia, Fausto Giunchiglia, Marco Pistore, Marco Roveri, Roberto Sebastiani, and Armando Tacchella. 2002. NuSMV 2: An OpenSource Tool for Symbolic Model Checking. In Proceedings of the 14th International Conference on Computer Aided Verification (CAV '02). Springer-Verlag, 359-364.

[17] Paul Clements and Linda Northrop. 2001. Software Product Lines: Practices and Patterns. Addison-Wesley Professional.

[18] Domenico Corapi. 2011. Nonmonotonic Inductive Logic Programming as Abductive Search. Ph.D. Dissertation. Imperial College London.

[19] Robert Darimont. 2014. GORE/KAOS in the industry: Lessons learnt. (2014) https://refsq.org/2014/2014/files/Robert-Darimont-GORE.pdf.

[20] Neil A. Ernst, Alexander Borgida, and Ivan Jureta. 2011. Finding incremental solutions for evolving requirements. In Proceedings of the IEEE 19th International Requirements Engineering Conference. 15-24.

[21] Pranav Garg, Christof Löding, P. Madhusudan, and Daniel Neider. 2014. ICE: A Robust Framework for Learning Invariants. In Proceedings of the 26th International Conference on Computer Aided Verification. 69-87.

[22] Martin Gebser, Roland Kaminski, Benjamin Kaufmann, and Torsten Schaub. 2014 Clingo = ASP + Control: Preliminary Report. CoRR abs/1405.3694 (2014).

[23] Martin Gebser, Benjamin Kaufmann, Roland Kaminski, Max Ostrowski, Torsten Schaub, and Marius Schneider. 2011. Potassco: The Potsdam Answer Set Solving Collection. AI Commun. 24, 2 (2011), 107-124.

[24] Carlo Ghezzi and Amir Molzam Sharifloo. 2013. Dealing with non-functional requirements for adaptive systems via dynamic software product-lines. In Software Engineering for Self-Adaptive Systems II. Springer, 191-213.

[25] Joel Greenyer, Daniel Gritzner, David Harel, and Assaf Marron. 2018. Towards Automated Defect Analysis Using Execution Traces of Scenario-Based Models. In Proceedings of the 6th International Conference on Model-Driven Engineering and Software Development MODELSWARD. 335-354.
[26] Jilles Van Gurp, Jan Bosch, and Mikael Svahnberg. 2001. On the Notion of Variability in Software Product Lines. In Proceedings of the Working IEEE/IFIP Conference on Software Architecture (WICSA '01). IEEE Computer Society, Washington, DC, USA, 45-.

[27] Mike Hinchey, Sooyong Park, and Klaus Schmid. 2012. Building Dynamic Software Product Lines. Computer 45, 10 (Oct. 2012), 22-26.

[28] Paola Inverardi and Marco Mori. 2011. Requirements models at run-time to support consistent system evolutions. In Requirements@Run.Time (RE@RunTime), 2011 2nd International Workshop on. IEEE, 1-8.

[29] Michael Jackson. 1995. Software Requirements and Specifications: A Lexicon of Practice, Principles and Prejudices. ACM Press/Addison-Wesley Publishing Co., New York, NY, USA.

[30] Shalinka Jayatilleke and Richard Lai. 2018. A systematic review of requirements change management. Information and Software Technology 93 (2018), 163 - 185.

[31] Ivan J. Jureta, Alexander Borgida, Neil A. Ernst, and John Mylopoulos. 2014. The Requirements Problem for Adaptive Systems. ACM Trans. Manage. Inf. Syst. 5, 3 (2014), 17:1-17:33.

[32] Ron Koymans. 1990. Specifying Real-time Properties with Metric Temporal Logic. Real-Time Syst. 2, 4 (1990), 255-299.

[33] Ron Koymans. 1992. Specifying message passing and time-critical systems with temporal logic. Vol. 651. Springer Science \& Business Media.

[34] Alexei Lapouchnian and John Mylopoulos. 2009. Modeling Domain Variability in Requirements Engineering with Contexts. In Proceedings of the 28th International Conference on Conceptual Modeling. 115-130.

[35] John W. Lloyd. 1987. Foundations of Logic Programming. Springer-Verlag.

[36] James Lockerbie, Neil Arthur Maiden, Jorgen Engmann, Debbie Randall, Sean Jones, and David Bush. 2012. Exploring the Impact of Software Requirements on System-wide Goals: A Method Using Satisfaction Arguments and I* Goal Modelling. Requir. Eng. 17, 3 (Sept. 2012), 227-254.

[37] Zohar Manna and Amir Pnueli. 1992. The Temporal Logic of Reactive and Concurrent Systems. Springer-Verlag.

[38] Stephen H. Muggleton and Christopher H. Bryant. 2000. Theory Completion Using Inverse Entailment. In Proceedings of 10th International Conference on Inductive Logic Programming. LNCS, Vol. 1866. 130-146.

[39] Chi Mai Nguyen, Roberto Sebastiani, Paolo Giorgini, and John Mylopoulos. 2016. Requirements evolution and evolution requirements with constrained goal models. In Proceedings of the 35th International Conference Conceptual Modeling. Springer, 544-552.

[40] Bashar Nuseibeh and Steve Easterbrook. 2000. Requirements Engineering: A Roadmap. In Proceedings of the Conference on The Future of Software Engineering (ICSE '00). ACM, New York, NY, USA, 35-46.

[41] Christophe Ponsard and Robert Darimont. 2017. Improving Requirements Engineering through Goal-oriented Models and Tools: Feedback from a Large Industrial Deployment. In Proceedings of the 12th International Conference on Software Technologies, ICSOFT. 372-381.

[42] SPF Santé Publique. 2013. Manuel belge de la régulation médicale. (2013). https://www.health.belgium.be/fr/manuel-belge-de-la-regulation-medicale

[43] RESPIRE. [n. d.]. ([n. d.]). https://www.dropbox.com/sh/wdew1sbcoqhhfe0/ AACBqCRj7epEyRX9teKYZ3bpa?dl=0.

[44] Siemens Products \& Services. 2018. Low Emission Zones - Reducing air pollution where it matters. (2018). https://new.siemens.com/global/en/products/mobility/ road-solutions/enforcement-and-tolling-solutions/low-emission-zone.html.

[45] Lightfoot Solutions, Lis Nixon Associates, and Baker Tilly. 2009. Efficiency review of the Welsh Ambulance Services NHS Trust. Technical Report. http://www.wales.nhs.uk/document/171903.

[46] Daniel Sykes, Domenico Corapi, Jeff Magee, Jeff Kramer, Alessandra Russo, and Katsumi Inoue. 2013. Learning Revised Models for Planning in Adaptive Systems. In Proceedings of the 2013 International Conference on Software Engineering. IEEE Press, 63-71.

[47] Amos Tversky. 1977. Features of similarity. Psychological Review 84, 4 (1977), 327--35.

[48] Axel van Lamsweerde. 2009. Requirements Engineering: From System Goals to UML Models to Software Specifications (1st ed.). Wiley Publishing.

[49] Pamela Zave and Michael Jackson. 1997. Four Dark Corners of Requirements Engineering. ACM Transactions on Software Engineering and Methodology 6, 1 (Jan. 1997), 1-30. 